patients can collect their own blood samples and mail them to the laboratory, removing the need for specialist equipment and skills at this stage.

Included in this study were 205 male volunteers (median age 70 years, range $41-75$ years) with no history of prostate disease. A venous blood sample was taken from each participant for the standard serum PSA test, and a drop of capillary blood was collected from the finger $(30 \mu l)$ for the new method; both tests were performed at the same laboratory.

The thresholds for positive tests were $>3 \mathrm{ng} / \mathrm{ml}$ for the standard PSA test and $>78 \mathrm{pg} / \mathrm{ml}$ for the nanotest (this threshold provides 100\% sensitivity). There were 24 (11.7\%) of 205 falsepositive results recorded with the nanotest. Results of the two tests correlated strongly $(r=0.98, P<0.001)$ with good agreement between the data sets up to values of $5 \mathrm{ng} / \mathrm{ml}$, above which the reliability of nanotest results was lost.

The authors conclude that, given the good correlation between methods, economic studies are now warranted to prove costeffectiveness of the nanotest, with a longterm view of adopting it for mass screening programs for prostate cancer.

Original article Azzouzi AR et al. (2007) Relevance of the prostate-specific antigen (PSA) nanotest compared to the classical PSA test in the organized mass screening of prostate cancer. BJU Int 99: 762-764

\section{Brachytherapy plus EBRT reduces the risk of ECE in prostate cancer patients}

Prostate brachytherapy is widely used to treat patients with low-risk prostate cancer, but its use in patients at high risk of extracapsular cancer extension (ECE) is controversial. Dattoli and colleagues investigated the long-term biochemical control rates when external beam radiotherapy (EBRT) and palladium-103-labelled $\left({ }^{103} \mathrm{Pd}\right)$ brachytherapy were used in combination between 1992 and 1996 to treat 243 consecutive patients (median age 66 years, range 43-88 years) who had at least one high-risk factor for ECE.

Participants were eligible if they had $\geq 1$ of the following risk factors: Gleason score $\geq 7$; serum PSA level $>10 \mathrm{ng} / \mathrm{ml}$; or prostatic acid phosphatase level $>2.5 \mathrm{U}$. Patients received
41 Gy EBRT, followed by a ${ }^{103} \mathrm{Pd}$ boost 4 weeks later. Follow-up was scheduled at 3, 6 and 12 months, then every 6-12 months thereafter. The researchers defined freedom from biochemical failure as serum PSA $\leq 0.2 \mathrm{ng} / \mathrm{ml}$ at last follow-up visit.

More than 5 years' follow-up was achieved in 198 patients (median 9 years, range 1-13 years). At 13 years' follow-up, the overall freedom from biochemical failure rate was 81\%; 41 patients experienced failure. The absolute risk of biochemical failure decreased over time to $1 \%$ after 6 years. Of all failures, 27 occurred within the first 3 years after treatment. The strongest predictor of failure was prostatic acid phosphatase level.

The authors conclude that the high tumor control rates achieved by brachytherapy, in combination with EBRT, has useful and durable effects for patients with prostate cancer at high risk of ECE.

Original article Dattoli M et al. (2007) Long-term prostate cancer control using palladium-103 brachytherapy and external beam radiotherapy in patients with a high likelihood of extracapsular cancer extension. Urology 69: 334-337

\section{Once-weekly oral alendronate prevents bone loss in men receiving ADT for prostate cancer}

A new study has shown that once-weekly oral bisphosphonate can prevent and improve bone loss in men undergoing androgen deprivation therapy (ADT) for prostate cancer.

In a randomized, double-blind, placebocontrolled, partial cross-over trial, 112 men with nonmetastatic prostate cancer who were receiving ADT were given either alendronate (70 mg once weekly) or placebo, together with calcium and vitamin D supplements. Overall, $39 \%$ of men had osteoporosis and $52 \%$ had low bone mass at baseline. After 1 year, men who received alendronate showed an increase in bone mineral density of $3.7 \%$ at the spine and $1.6 \%$ at the femoral neck, compared with losses of $1.4 \%$ and $0.7 \%$, respectively, in the placebo group-a difference of $5.1 \%$ at the spine $(P<0.001)$ and $2.3 \%$ at the femoral neck $(P<0.001)$. Patients treated with ADT and alendronate also had significant and clinically important decreases in all markers of bone turnover compared with those who received ADT plus placebo. 